\title{
RELATIONSHIP BETWEEN SERUM \\ 25-HYDROXYVITAMIN D CONCENTRATION AND ACUTE INFLAMMATORY MARKERS IN HOSPITALIZED PATIENTS WITH SARS-COV-2 INFECTION
}

\author{
Teodoro J. Oscanoa ${ }^{1,2}{ }^{(0}$, José Amado ${ }^{1,2}{ }^{\infty}$, Rawia A. Ghashut ${ }^{3}$, Roman Romero-Ortuno ${ }^{4,5}$ \\ ${ }^{1}$ Faculty of Medicine, Universidad Nacional Mayor de San Marcos, Lima, Perú \\ ${ }^{2}$ Faculty of Medicine, Universidad de San Martín de Porres, Drug Safety Research Centre, Hospital Almenara, ESSALUD, Lima, Perú \\ ${ }^{3}$ Academic Unit of Anaesthesia, College of Medical, Veterinary and Life of Sciences, University of Glasgow, United Kingdom \\ ${ }^{4}$ Discipline of Medical Gerontology, Mercer's Institute for Successful Ageing, St James's Hospital, Dublin, Ireland \\ ${ }^{5} \mathrm{Global}$ Brain Health Institute, Trinity College, Dublin, Ireland
}

\begin{abstract}
INTRODUCTION: There is experimental and clinical evidence that the serum concentration of 25-hydroxyvitamin $D[25(\mathrm{OH}) \mathrm{D})]$ may decrease in acute systemic inflammatory responses; in this context, low values may not necessarily indicate a pre-existing deficiency. This may also apply to low 25(OH)D levels found in the context of the systemic inflammatory response caused by SARS-CoV- 2 infection.
\end{abstract}

To conduct a systematic review of the relationship between serum 25(OH)D and the concentrations of C-reactive protein (CRP), interleukin 6 (IL-6) and tumour necrosis factor $\alpha$ (TNF- $\alpha$ ) in acutely hospitalized patients with SARS-CoV-2 infection.

MATERIAL AND METHODS: We searched PubMed, EMBASE, Google Scholar and the Cochrane Database of Systematic Reviews for studies published between January 2020 and February 2021. In each study, the authors compared levels of inflammatory markers between patients reported as having low levels of 25(OH) $D$ and those above the study cut-off.

RESULTS: 18 studies were included ( $n=3482$, mean age $63.5 \pm 9.3$ years, $56.9 \%$ men). The cut-off for the definition of low $25(\mathrm{OH})$ D varied across studies. In all studies, mean values for inflammatory markers were higher in the low 25(OH)D groups. These differences were statistically significant $(p<0.05)$ in $6 / 15$ studies with CRP, 4/8 with IL- 6 and 0/1 with TNF- $\alpha$.

CONCLUSIONS: Markers of acute systemic inflammatory response were elevated in patients with SARSCoV-2 infection and low concentrations of 25(OH)D. Therefore, the vitamin D status in those patients should be interpreted with caution, and studies should be designed to assess whether hypovitaminosis $D$ could be an epiphenomenon.

KEY WORDS: SARS-CoV-2, COVID-19, interleukin 6, C-reactive protein, vitamin D, 25-hydroxyvitamin D 


\section{INTRODUCTION}

The COVID-19 pandemic has by March 2021 caused more than 2.7 million deaths worldwide [1] and forced medical science not only to create vaccines at a pace never seen before but also to study risk factors, especially modifiable ones, to reduce the risk of severe or fatal forms of SARS-CoV-2 infection. One of the studied modifiable risk factors has been vitamin $\mathrm{D}$ status.

To date, four systematic and meta-analytical studies have analysed the issue and have concluded that there is a probable cause-and-effect association between low concentrations of 25-hydroxyvitamin $\mathrm{D}[25(\mathrm{OH}) \mathrm{D}]$ and increased mortality and severity due to SARS-CoV-2 infection [2-5]. Scientific controversy has arisen for three reasons: first, because there is experimental and clinical evidence before the COVID-19 pandemic, that acute inflammation can cause a reduction in $25(\mathrm{OH}) \mathrm{D}$ concentrations, in patients who previously had normal concentrations, for which the evaluation of the status of this vitamin should be taken with caution in this scenario [6-8]. Second, because the studies on which the metanalytical studies were based did not determine baseline concentrations of 25(OH)D before infection or hospital admission for severe or fatal forms of SARS-CoV-2 infection. Szeto et al. (2020) recently published a study where they determined the serum concentration of 25(OH)D in patients with SARS-CoV-2 infection before being hospitalized and found no relationship between 25(OH)D concentration and discharge status, mortality, length of stay, intubation status, or renal replacement therapy [9]. Third, a meta-analytic study that analysed clinical trials in which vitamin $D_{3}$ was administered in patients with SARS-CoV-2 infection and hypovitaminosis D, did not reduce mortality [10].

To further evaluate the association between high acute illness severity and vitamin D status in SARS-CoV-2 patients, the authors conducted a systematic review of the relationship between serum $25(\mathrm{OH}) \mathrm{D}$ and the concentration of serum markers of acuity of illness such as C-reactive protein (CRP), tumour necrosis factor $\alpha$ (TNF- $\alpha$ ) and interleukin 6 (IL-6) in patients acutely hospitalized with SARS$\mathrm{CoV}-2$ infection. Those three markers were selected because it has been experimentally reported in humans that their elevation may be associated with a decrease of the concentration of previously normal 25(OH)D [11].

\section{MATERIAL AND METHODS}

This study was conducted following the guidelines of the Preferred Reporting Items for Systematic Reviews and Meta-analyses (PRISMA) [12].

\section{Search strategy}

Two independent investigators performed a systematic search in PubMed, EMBASE, Google Scholar and the Cochrane Database of Systematic Reviews for studies published between January 2020 and February 2021. In addition, a secondary search based on the reference lists of retrieved articles was conducted. The following combined search terms were used in PubMed: ('Novel coronavirus' or 'Coronavirus disease 2019' or 'Corona-virus 2019' or 'nCoV-2019' or '2019-nCoV' or 'COVID-19' or 'SARS-CoV-2' or '25OHD' or 'acute-phase proteins' or 'C-reactive protein' or 'tumour necrosis factor (TNF) $-\alpha$ ' or 'interleukin (IL) -6'.

\section{Eligibility criteria}

We searched for randomized controlled trials (RCTs) or observational studies reporting data on serum 25(OH)D concentration and SARS-CoV-2 infection severity or mortality. Studies in English or other languages meeting the following criteria were included: a) acutely hospitalized COVID-19 patients aged 18 or more years who were diagnosed according to the interim guidance of the World Health Organization [13]; b) inclusion of the median or mean and standard deviation for laboratory test values of 25(OH)D, and sample size with information on demographics, comorbidities and complications; $c$ ) the study presented data on serum CRP, TNF- $\alpha$ and/or IL-6. The following exclusion criteria were applied: reviews, abstracts, discussion summaries, and insufficient reported data.

\section{Quality assessment}

The quality of observational studies (cohort and case-control studies) and RCTs were assessed according to the Newcastle-Ottawa Quality Assessment Scale (NOS) [14] and the Cochrane Risk of Bias Assessment Tool [15], respectively. Two investigators evaluated the quality of the studies independently. Conflicting results were resolved by discussion and involvement of a third reviewer if necessary.

\section{Data extraction}

The following data were extracted from each study: authors, study location, year of publication, 


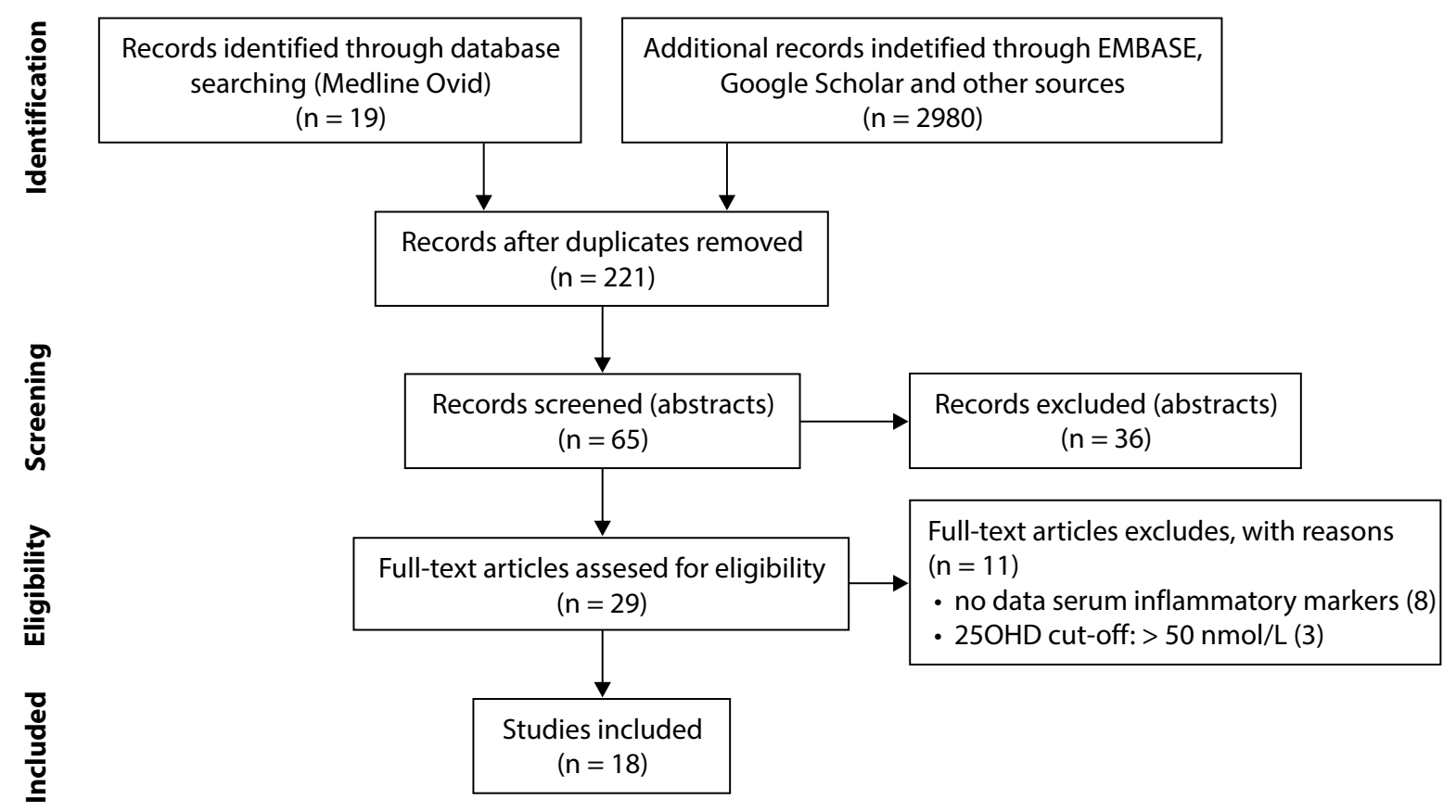

FIGURE 1. Flowchart of included studies

study design, number of participants, sex, age at baseline, serum 25(OH)D concentration, and concentration of CRP, TNF- $\alpha$ and IL-6. Even though some studies have considered other $25(\mathrm{OH}) \mathrm{D}$ cutoff values [16], where possible 25(OH)D deficiency was considered as $<50 \mathrm{nmol} / \mathrm{L}(<20 \mathrm{ng} / \mathrm{ml})$ [17]. In each study, concentrations of inflammatory markers between patients reported as having low concentrations of $25(\mathrm{OH}) \mathrm{D}$ and those above the study cut-off were compared. The p-value resulting from the comparison between groups of patients with and without low concentrations of 25(OH)D was calculated with the 2-sided Mann Whitney $\mathrm{U}$ test.

\section{RESULTS}

After screening 2999 citations, 18 observational eligible studies (cohort, 10; case-control, 10; cross-sectional, 4) were included (Fig. 1), combining to a total sample of 3482 participants. The characteristics of included studies are summarized in Table 1. The studies were from Austria [18], Germany [19], China [20], Greece [21], India [22, 23], Iran [24], Italy [25-27], Spain [28], Turkey [29-31], UK [32-34], and USA [35].

Overall, the mean age of the participants was $63.5 \pm 9.3$ years and $56.9 \%$ were men. The mean NOS score of included studies was 8 (range: 7-9).
The outcomes reported in the included studies are presented in Table 1.

All measurements of inflammatory markers were performed during hospitalization, and none of the studies measured serum 25(OH)D concentrations before hospitalization. In all 18 studies, patients with low 25(OH)D had elevated concentrations of CRP, IL- 6 and/or TNF- $\alpha$. These differences were statistically significant $(p<0.05)$ in $6 / 15$ studies with CRP, 4/8 with IL-6 and 0/1 with TNF- $\alpha$ (Tab. 2).

\section{DISCUSSION}

The present study on the relationship between $25(\mathrm{OH}) \mathrm{D}$ and markers of systemic inflammatory response in SARS-CoV-2 infection found elevated concentrations of CRP, IL- 6 and/or TNF- $\alpha$ in patients with low concentrations of 25(OH)D. In the context of a patient with SARS-CoV-2 infection, the present finding can be interpreted in two ways; first, that there may be a relationship between the low concentrations of $25(\mathrm{OH}) \mathrm{D}$ and the acute inflammatory process (as evidenced by elevations of CRP, IL- 6 and/or TNF- $\alpha$ ), which could be confounded as the low concentrations of $25(\mathrm{OH}) \mathrm{D}$ being associated with greater SARS-CoV-2 severity or mortality. Second, that the acute inflammatory process may induce a reduction in the concentration of 25(OH)D in patients who prior to infection 


\begin{tabular}{|c|c|c|c|c|c|c|c|c|c|c|c|}
\hline$\stackrel{\check{0}}{z}$ & a & $\wedge$ & $\sigma$ & $\sigma$ & の & $\infty$ & $\sigma$ & a & $\infty$ & $\wedge$ & $\wedge$ \\
\hline 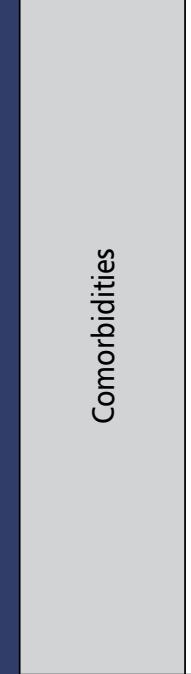 & 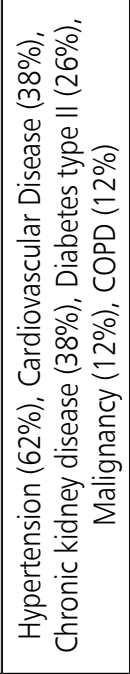 & 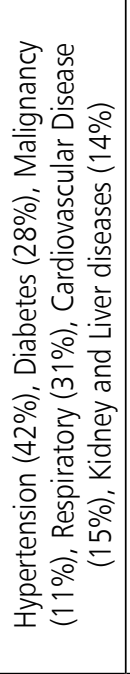 & 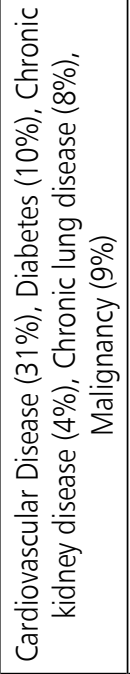 & 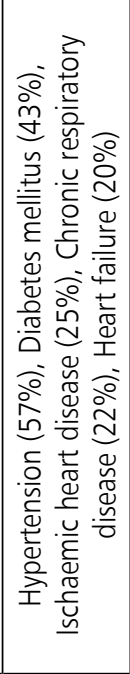 & 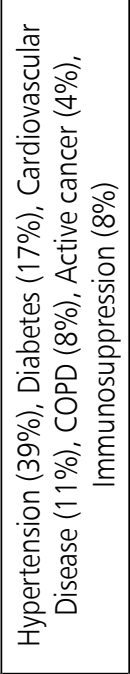 & $\frac{⿱}{z}$ & 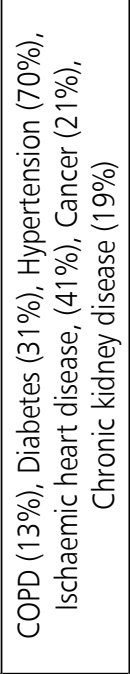 & 兴 & 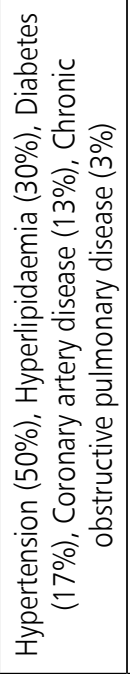 & 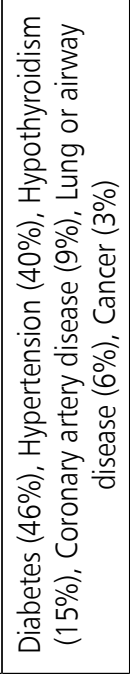 & 愛 \\
\hline 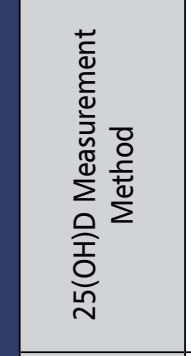 & 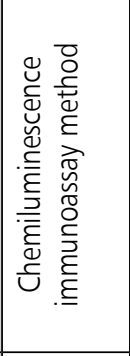 & $\frac{a}{z}$ & 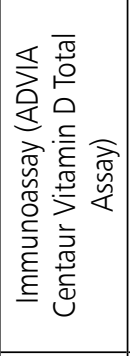 & $\frac{⿱ 亠 乂}{z}$ & 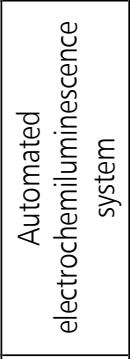 & 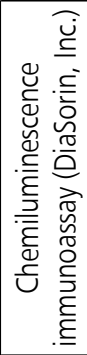 & 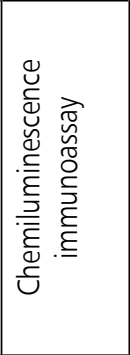 & 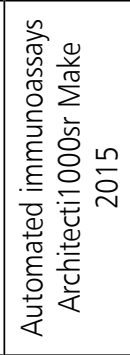 & 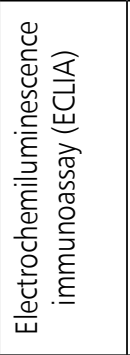 & 魚 & 受 \\
\hline 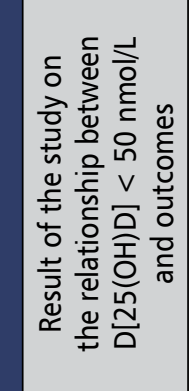 & 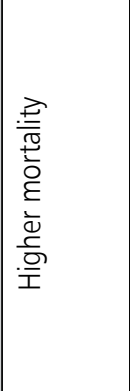 & 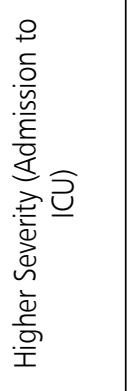 & 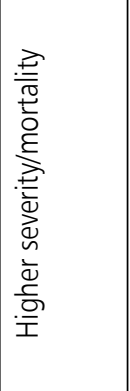 & 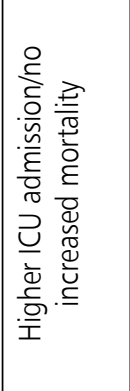 & 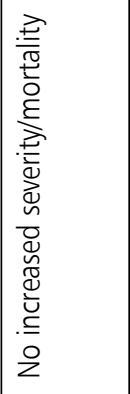 & 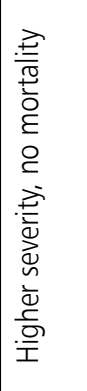 & 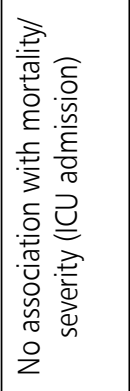 & 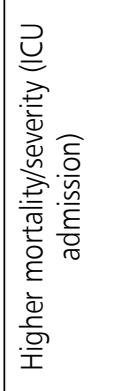 & 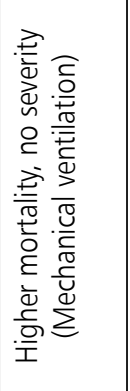 & 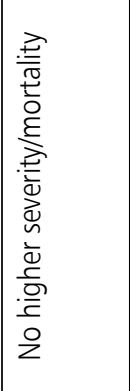 & 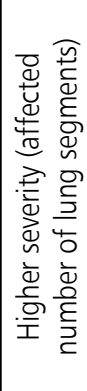 \\
\hline 胥 & 缉 & $\begin{array}{l}n \\
\infty \\
0 \\
0\end{array}$ & 8 & $\frac{m}{\infty}$ & $\overline{6}$ & 吕 & $\curvearrowright$ & $\begin{array}{l}\stackrel{7}{*} \\
\stackrel{\text { ก }}{n}\end{array}$ & 능 & in & $\frac{\stackrel{n}{7}}{\dot{J}}$ \\
\hline 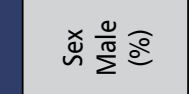 & $\bar{\lambda}$ & ळें & $\bar{n}$ & 8 & 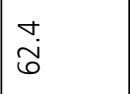 & $\stackrel{\sim}{\mathscr{f}}$ & $\underset{\dot{m}}{\stackrel{m}{n}}$ & $\begin{array}{l}\ddot{\theta} \\
\ddot{6} \\
\ddot{6}\end{array}$ & $\infty$ & ஜீ & 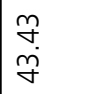 \\
\hline $\begin{array}{l}\frac{0}{0} \\
\sum_{\tilde{N}}^{\circ} \\
\stackrel{N}{n}\end{array}$ & $\mathscr{F}$ & $\stackrel{ \pm}{m}$ & $\stackrel{\stackrel{\infty}{\infty}}{\infty}$ & $\stackrel{\circ}{\curvearrowright}$ & 으 & $\stackrel{\stackrel{n}{m}}{m}$ & $\stackrel{\text { I }}{\simeq}$ & $\stackrel{\text { 莒 }}{\sim}$ & $\stackrel{m}{ }$ & $\stackrel{\circ}{\gamma}$ & $\stackrel{\approx}{\approx}$ \\
\hline 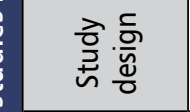 & $\cup$ & 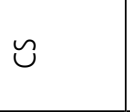 & $\cup$ & $\cup$ & $\cup$ & $\tilde{U}$ & $\cup$ & $\cup$ & $\cup$ & 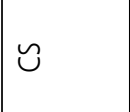 & $\cup$ \\
\hline 辛 & $\begin{array}{l}\text { 离 } \\
\pm\end{array}$ & 兰 & 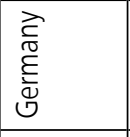 & 兰 & 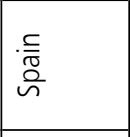 & : & 离 & $\begin{array}{l}\frac{0}{0} \\
\underline{\underline{\underline{O}}}\end{array}$ & 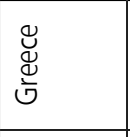 & $\frac{-\frac{0}{0}}{\underline{\underline{I}}}$ & 离 \\
\hline 产 & 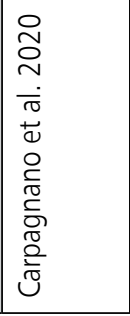 & 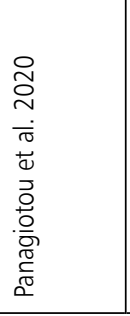 & 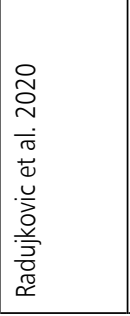 & 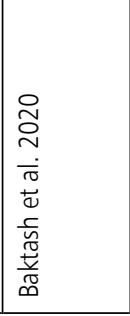 & 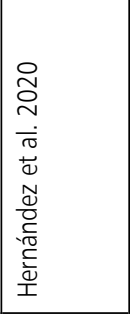 & 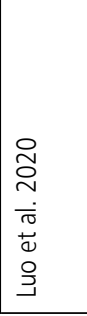 & 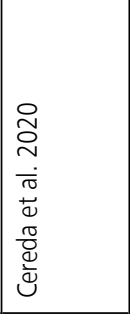 & 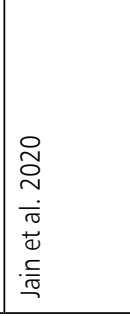 & 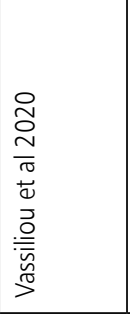 & 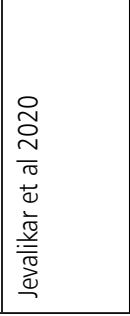 & 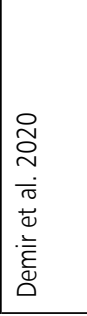 \\
\hline
\end{tabular}




\begin{tabular}{|c|c|c|c|c|c|c|c|}
\hline O) & $\wedge$ & $\infty$ & $\infty$ & $\infty$ & $r$ & $r$ & $\infty$ \\
\hline 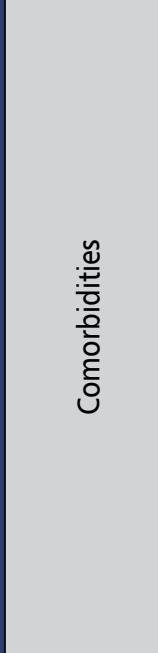 & 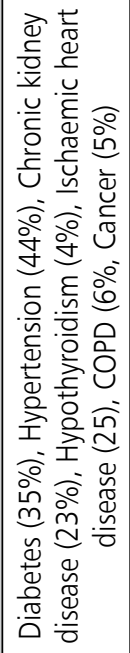 & 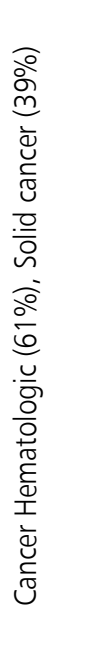 & 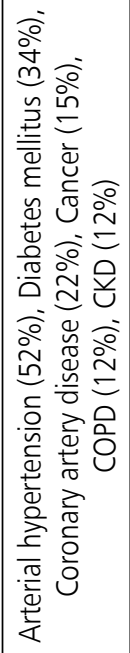 & 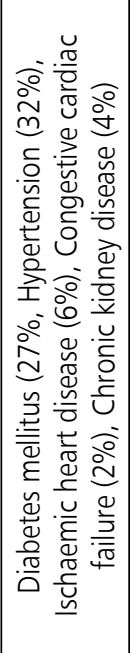 & 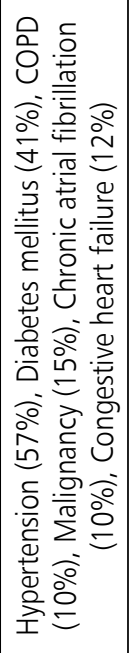 & 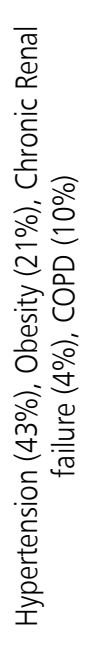 & 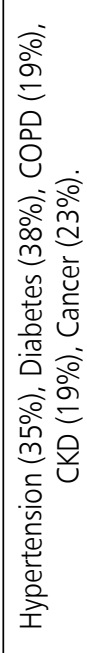 \\
\hline 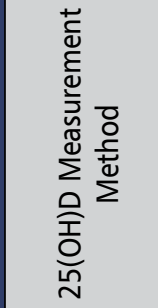 & $\frac{o}{z}$ & 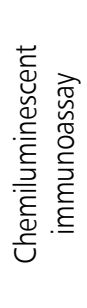 & 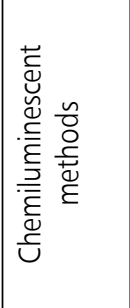 & $\stackrel{\propto}{z}$ & $\stackrel{\frac{\alpha}{z}}{ }$ & $\frac{o}{z}$ & $\stackrel{\propto}{z}$ \\
\hline 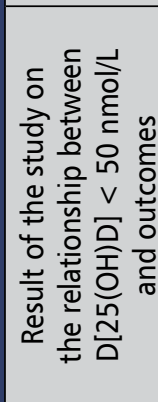 & 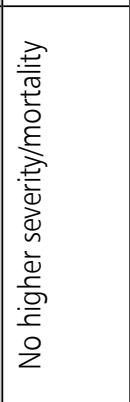 & 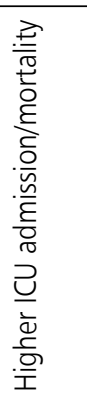 & 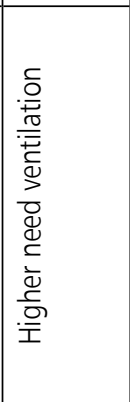 & 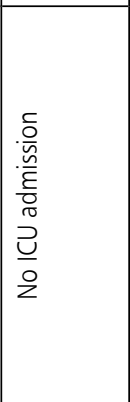 & 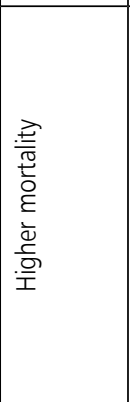 & 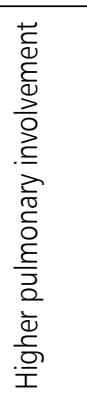 & 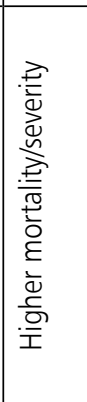 \\
\hline 丞 & हิ & $\overline{6}$ & ஜூ & in & ñ. & $\stackrel{\text { n? }}{\stackrel{2}{\aleph}}$ & $\stackrel{\sim}{\infty}$ \\
\hline 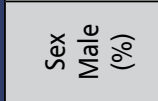 & \begin{tabular}{|l|}
$\underline{n}$ \\
mi \\
$m$
\end{tabular} & $\bullet$ & $\begin{array}{l}\stackrel{0}{\leftrightarrow} \\
\text { ñ }\end{array}$ & $\stackrel{\infty}{\stackrel{\infty}{n}}$ & 离 & \begin{tabular}{|l|}
$\infty$ \\
0 \\
$\infty$ \\
$\dot{\sigma}$ \\
\end{tabular} & กุ' \\
\hline$\underset{\substack{\frac{0}{0} \\
\stackrel{n}{n}}}{\stackrel{N}{n}}$ & 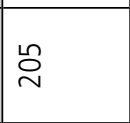 & $\stackrel{\infty}{=}$ & $\stackrel{\infty}{m}$ & $\bar{\gamma}$ & $\underset{g}{\stackrel{g}{9}}$ & ก & ○ั \\
\hline 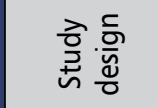 & 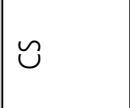 & $\cup$ & $\cup$ & $\cup$ & $\cup$ & $\cup$ & $\cup$ \\
\hline 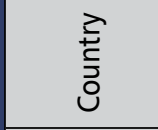 & 胥 & $\frac{.0}{\frac{D}{5}}$ & 空 & 弚 & $\begin{array}{l}\text { बे } \\
\stackrel{\overrightarrow{⿱ 亠 䒑}}{\equiv}\end{array}$ & 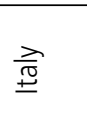 & 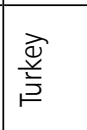 \\
\hline 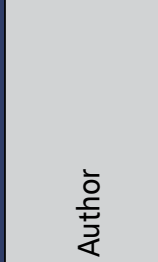 & 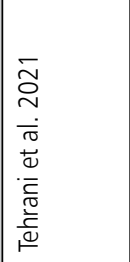 & 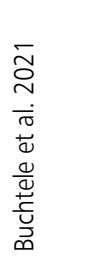 & 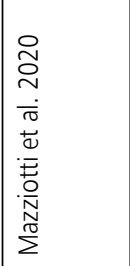 & 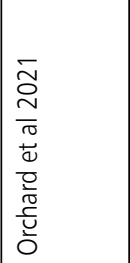 & 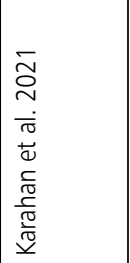 & 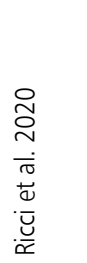 & 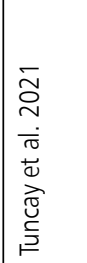 \\
\hline
\end{tabular}




\begin{tabular}{|c|c|c|c|c|c|}
\hline Study & $\begin{array}{l}\text { 25-hydroxyvitamin } \\
\text { (nmol/L) }\end{array}$ & $\begin{array}{c}\text { Size } \\
\text { sample }\end{array}$ & $\begin{array}{c}\mathrm{CRP}(\mathrm{mg} / \mathrm{L}) \\
\text { (mean/median) }\end{array}$ & $\begin{array}{l}\text { Interleukin-6, pg/mL } \\
\text { (mean/median) }\end{array}$ & $\begin{array}{l}\text { Serum TNF } \alpha \text { in } \mathrm{pg} / \mathrm{mL} \\
\text { (mean/median) }\end{array}$ \\
\hline \multirow[t]{2}{*}{ Carpagnano et al. 2020} & $<25$ & 10 & $102 \pm 79.98$ & $244 \pm 468.35$ & NR \\
\hline & $>50$ & 11 & $91 \pm 41.74$ & $83 \pm 44.39$ & NR \\
\hline \multirow[t]{2}{*}{ Panagiotou et al. 2020} & $33.5 \pm 16.8$ & 42 & $143.4 \pm 99.4$ & NR & NR \\
\hline & $48.1 \pm 38.2$ & 92 & $107.9 \pm 92.0^{*}$ & NR & \\
\hline \multirow[t]{2}{*}{ Radujkovic et al. 2020} & $\leq 30$ & 29 & $N R$ & $70.5(32.0-326.3)$ & $N R$ \\
\hline & $>30$ & 64 & NR & $29.7(14.3-59.9)^{*}$ & NR \\
\hline \multirow[t]{2}{*}{ Baktash et al. 2020} & $\leq 30$ & 39 & $191(108-274)$ & NR & NR \\
\hline & $>30$ & 31 & $155((96-252)$ & NR & NR \\
\hline \multirow[t]{2}{*}{ Hernández et al. 2020} & $<50$ & 162 & $61(31-136)$ & $58.9(19.1-124.0)$ & NR \\
\hline & $>50$ & 35 & $32(23-87)$ & $45.6(20.5-119.0)$ & NR \\
\hline \multirow[t]{2}{*}{ Luo et al. 2020} & $23.1(18.1-28.3)$ & 74 & $64.5(12.3-422)$ & $9.33(2.4-28.0)$ & NR \\
\hline & $27.5(21.8-34.5)$ & 261 & $12(5.5-30.0)^{* *}$ & $2.1(1.5-4.1) * *$ & NR \\
\hline \multirow[t]{2}{*}{ Cereda et al. 2020} & $<50$ & 99 & $115(55.6-171.4)$ & NR & NR \\
\hline & $\geq 50$ & 30 & $68.1(40-143.9)$ & NR & NR \\
\hline \multirow[t]{2}{*}{ Jain et al. 2020} & $<50$ & 90 & NR & $19.34 \pm 6.17$ & $13.26 \pm 5.64$ \\
\hline & $>50$ & 64 & NR & $12.18 \pm 4.29^{*}$ & $11.87 \pm 3.15$ \\
\hline \multirow[t]{2}{*}{ Vassiliou et al 2020} & $<38$ & 15 & $190(50-260)$ & NR & NR \\
\hline & $>38$ & 15 & $100(40-170)$ & NR & NR \\
\hline \multirow[t]{2}{*}{ Jevalikar et al 2020} & $<50$ & 197 & $62.2 \pm 343.7$ & $46.3 \pm 113.5$ & $N R$ \\
\hline & $>50$ & 212 & $45.1 \pm 56.0$ & $45 \cdot 9 \pm 121$ & $N R$ \\
\hline \multirow[t]{2}{*}{ Demir et al. 2020} & $<25$ & 99 & $22.49 \pm 2.88$ & NR & NR \\
\hline & $>50$ & 42 & $10.99 \pm 4.10^{* *}$ & NR & NR \\
\hline \multirow[t]{2}{*}{ Tehrani et al. 2021} & $<25$ & 25 & $4.34 \pm 2.17$ & NR & \\
\hline & $>50$ & 88 & $3.55 \pm 1.7^{*}$ & NR & \\
\hline \multirow[t]{2}{*}{ Buchtele et al. 2021} & $<30$ & 96 & $233(76-334)$ & $N R$ & \\
\hline & $>50$ & 47 & $184(62-408)$ & NR & \\
\hline \multirow[t]{2}{*}{ Mazziotti et al. 2020} & $<30$ & 161 & $105(10-2270)$ & $74.0(4-1573)$ & $N R$ \\
\hline & $>50$ & 36 & $60(10-210)$ & $22.0(8-451)$ & NR \\
\hline \multirow[t]{2}{*}{ Orchard et al 2021} & $<50$ & 41 & $167(120-221)$ & NR & NR \\
\hline & $>50$ & 9 & $147(116-242)$ & NR & NR \\
\hline \multirow[t]{2}{*}{ Karahan et al. 2021} & $26 \pm 16.0$ & 47 & $108.7 \pm 78.3$ & NR & NR \\
\hline & $65.75 \pm 21$ & 102 & $44.2 \pm 59.5^{* *}$ & NR & NR \\
\hline \multirow[t]{2}{*}{ Ricci et al. 2020} & $<25$ & 22 & $N R$ & $85.2 \pm 78.01$ & NR \\
\hline & $\geq 25$ & 30 & NR & $39.6 \pm 17.87^{* *}$ & NR \\
\hline \multirow[t]{2}{*}{ Tuncay et al. 2021} & $26.25 \pm 3.44$ & 26 & $14.1 \pm 9$ & NR & $N R$ \\
\hline & $52.93 \pm 12.83$ & 450 & $4.4 \pm 1$ ** & NR & $N R$ \\
\hline
\end{tabular}

${ }^{*} p<0.05 ;{ }^{* *} p<0.001 ; \mathrm{NR}-$ not reported

may have had a normal 25(OH)D level. Therefore, low concentrations of 25(OH)D reported in the included studies may represent an epiphenomenon of the acute inflammatory process associated with SARS-CoV-2 infection, especially in its severe forms.

Before the pandemic, there was experimental evidence in animals [36] and humans that the acute in- 
flammatory process can induce a reduction in serum $25(\mathrm{OH}) \mathrm{D}$ concentrations in a secondary way [11]. Smolders et al. investigated whether systemic inflammation lowers circulating 25(OH)D concentrations using the experimental human endotoxemia model (bolus of $E$. Coli-derived lipopolysaccharide, LPS), and they found a significant decrease in 25(OH)D concentrations 2-3 hours after infusion, compared to baseline concentrations [11]. On the other hand, the fall in the concentrations of 25(OH)D coincided with an increase in the concentrations of proinflammatory cytokines TNF- $\alpha$, IL- 6 , and IL-8, and 25(OH)D concentrations recovered to baseline 6 hours following cessation of LPS infusion. In a systematic study, Silva et al. found that patients with normal total concentrations of $25(\mathrm{OH}) \mathrm{D}$ undergo a significant reduction in those levels when facing an acute injury [6].

Acute insults where this phenomenon of acute reduction in total $25(\mathrm{OH}) \mathrm{D}$ concentration occur are knee/hip arthroplasty [37-39], acute myocardial infarction [40], acute pancreatitis [41], first dose IV bisphosphonate [42], and cardiopulmonary bypass surgery [43]. In all the studies included in the systematic study, CRP was elevated. The recovery of the basal concentrations took 2 weeks, although in 2 studies it took up to 90 days $[38,40]$. In two studies, the concentration of a total of $25(\mathrm{OH}) \mathrm{D}$ was not modified, but the baseline sample was taken on the second day of the event [6]. Hypothetically, in some cases of SARS-CoV-2 infection, patients with previously normal 25(OH)D concentrations, after several days of the onset of the disease, may go to hospitals where low concentrations of $25(\mathrm{OH}) \mathrm{D}$ are detected, but this may be induced by the acute inflammatory process. It should be noted that the magnitude of the decrease in concentrations of $25(\mathrm{OH}) \mathrm{D}$ could depend on other unknown factors, which should be investigated.

The mechanisms by which the acute inflammatory process may induce a decrease in $25(\mathrm{OH}) \mathrm{D}$ are unknown, but it has been hypothesized that the contributing factors are acute stress haemodilution, interstitial extravasation, decreased synthesis of binding proteins, and renal wasting of $25(\mathrm{OH})$ $D$ [44]. The methods to discern if a reduction in 25(OH)D may be induced by an acute inflammatory state are - apart from the baseline measurement that is not feasible in COVID-19, the correction according to CRP concentrations and albumin $[8,38$, $45]$ or the determination of $25(\mathrm{OH}) \mathrm{D}_{3}$ in hair [46]. The correction for IL-6 concentrations has been re- ported for the correction of the measurement of concentrations of iron, zinc and selenium, which also decrease in an acute inflammatory process [47]. Studies before the pandemic have recommended taking the results of a patient's vitamin D status with caution if they have a CRP $>10 \mathrm{mg} / \mathrm{L}$ and albumin concentrations > $3.5 \mathrm{~g} / \mathrm{dL}$ [8]. On the other hand, it should be taken into account that establishing a cause-effect relationship between severity/mortality of COVID-19 and vitamin D, solely from measurements of 25(OH)D is difficult because to know exactly the vitamin $D$ status of a patient requires measurement of not only 25(OH)D (circulating reservoir), but also of 1,25-dihydroxy vitamin $D$ and vitamin $\mathrm{D}$ binding protein (VDBP). Approximately $85 \%$ of VDBP binds total circulating vitamin D [48]. The non-VDBP fraction (bioavailable vitamin D) consists primarily of albumin-bound (14\%), leaving the remainder in the free form (less than 1\%) [49]. The design of new studies is necessary to know in detail the relationship between vitamin D status and the severity/mortality of COVID-19.

Butler-Laporte et al. recently published a study using Mendelian randomization with genetic variants strongly associated with 25(OH)D levels in a genome-wide association study (GWAS) of 443.734 participants of European ancestry, and they did not observe evidence to support an association between 25(OH)D levels and COVID-19 susceptibility, severity, or hospitalization [50]. It is interesting that the findings of the present systematic study and the results of the described 2-sample Mendelian randomization study carried out with different methods, contribute with the evidence according to which there would not be a causal relationship between decreased 25(OH)D levels and severity of COVID-19.

\section{Limitations}

The present study has the limitation of being based on observational studies and although 15 studies reported inflammation markers such as CRP concentrations, only 10 and one studies reported measurement of IL- 6 and TNF- $\alpha$, respectively, and some studies had different cut-off points for 25(OH)D levels. Another limitation is the lack of a comparison group (i.e. Vitamin D status in patients with mild illness or normal concentrations of proinflammatory cytokines). In addition, as stated above, none of the included studies had ascertained Vitamin D concentrations before hospital admission. 


\section{CONCLUSIONS}

In conclusion, the present study found that markers of the acute systemic inflammatory response are elevated in patients with low concentrations of 25(OH)D and with SARS-CoV-2 infection. Therefore, the results of the vitamin $D$ status in these patients should be taken with caution, and studies should be designed to assess whether hypovitaminosis $D$ could be an epiphenomenon of the systemic inflammatory response.

\section{REFERENCES}

1. Johns Hopkins University. The Grants Register 2021. 2020: 496-496, doi: 10.1057/978-1-349-95988-4 521.

2. Pereira M, Dantas Damascena A, Galvão Azevedo LM, et al. Vitamin $D$ deficiency aggravates COVID-19: systematic review and meta-analysis. Crit Rev Food Sci Nutr. 2020 [Epub ahead of print]: 1-9, doi: 10.1080/10408398.2020.1841090, indexed in Pubmed: 33146028.

3. Liu N, Sun J, Wang $X$, et al. Low vitamin D status is associated with coronavirus disease 2019 outcomes: a systematic review and meta-analysis. Int J Infect Dis. 2021; 104: 58-64, doi: 10.1016/j. ijid.2020.12.077, indexed in Pubmed: 33401034.

4. Munshi $R$, Hussein MH, Toraih EA, et al. Vitamin D insufficiency as a potential culprit in critical COVID-19 patients. J Med Virol. 2021; 93(2): 733-740, doi: 10.1002/jmv.26360, indexed in Pubmed: 32716073.

5. Kazemi A, Mohammadi V, Aghababaee SK, et al. Association of Vitamin D Status with SARS-CoV-2 Infection or COVID-19 Severity: A Systematic Review and Meta-analysis. Adv Nutr. 2021 [Epub ahead of print], doi: 10.1093/advances/nmab012, indexed in Pubmed: 33751020.

6. Silva MC, Furlanetto TW. Does serum 25-hydroxyvitamin D decrease during acute-phase response? A systematic review. Nutr Res. 2015; 35(2): 91-96, doi: 10.1016/j.nutres.2014.12.008, indexed in Pubmed: 25631715.

7. Duncan A, Talwar D, McMillan DC, et al. Quantitative data on the magnitude of the systemic inflammatory response and its effect on micronutrient status based on plasma measurements. Am J Clin Nutr. 2012; 95(1): 64-71, doi: 10.3945/ajcn.111.023812, indexed in Pubmed: 22158726.

8. Ghashut RA, Talwar D, Kinsella J, et al. The effect of the systemic inflammatory response on plasma vitamin $25(\mathrm{OH}) \mathrm{D}$ concentrations adjusted for albumin. PLoS One. 2014; 9(3): e92614, doi: 10.1371/ journal.pone.0092614, indexed in Pubmed: 24667823.

9. Szeto B, Zucker JE, LaSota ED, et al. Vitamin D Status and COVID-19 Clinical Outcomes in Hospitalized Patients. Endocr Res. 2021; 46(2): 66-73, doi: 10.1080/07435800.2020.1867162, indexed in Pubmed: 33380209.

10. Shah K, Saxena D, Mavalankar D. Vitamin D supplementation, COVID-19 and disease severity: a meta-analysis. QJM. 2021; 114(3): 175-181, doi: 10.1093/qjmed/hcab009, indexed in Pubmed: 33486522 .
11. Smolders J, van den Ouweland J, Geven C, et al. Letter to the Editor: Vitamin D deficiency in COVID-19: Mixing up cause and consequence. Metabolism. 2021; 115: 154434, doi: 10.1016/j. metabol.2020.154434, indexed in Pubmed: 33217408.

12. Moher $D$, Liberati $A$, Tetzlaff J, et al. PRISMA Group, PRISMA Group, PRISMA Group, PRISMA Group, PRISMA Group, PRISMA Group, PRISMA Group. Preferred reporting items for systematic reviews and meta-analyses: the PRISMA Statement. Open Med. 2009; 3(3): e123-e130, indexed in Pubmed: 21603045.

13. Yadav A, Mohite S. A Review on Severe Acute Respiratory Infection (SARI) and its Clinical Management in Suspect/Confirmed Novel Coronavirus ( $\mathrm{nCoV}$ ) Cases. Research Journal of Pharmaceutical Dosage Forms and Technology. 2020; 12(3): 178, doi: 10.5958/09754377.2020.00030.0.

14. Stang A. Critical evaluation of the Newcastle-Ottawa scale for the assessment of the quality of nonrandomized studies in meta-analyses. Eur J Epidemiol. 2010; 25(9): 603-605, doi: 10.1007/s10654-0109491-z, indexed in Pubmed: 20652370.

15. Higgins JPT, Altman DG, Gøtzsche PC, et al. Cochrane Bias Methods Group, Cochrane Statistical Methods Group. The Cochrane Collaboration's tool for assessing risk of bias in randomised trials. BMJ. 2011; 343: d5928, doi: 10.1136/bmj.d5928, indexed in Pubmed: 22008217.

16. Benskin LL. A Basic Review of the Preliminary Evidence That COVID-19 Risk and Severity Is Increased in Vitamin D Deficiency. Front Public Health. 2020; 8: 513, doi: 10.3389/fpubh.2020.00513, indexed in Pubmed: 33014983.

17. World Medical Association (WMA). International Year Book and Statesmen's Who's Who. , doi: 10.1163/1570-6664_iyb_sim_org_38991.

18. Buchtele N, Lobmeyr E, Cserna J, et al. Prevalence and Impact of Vitamin D Deficiency in Critically III Cancer Patients Admitted to the Intensive Care Unit. Nutrients. 2020; 13(1), doi: 10.3390/nu13010022, indexed in Pubmed: 33374662.

19. Radujkovic A, Hippchen T, Tiwari-Heckler S, et al. Vitamin D Deficiency and Outcome of COVID-19 Patients. Nutrients. 2020; 12(9), doi: 10.3390/nu12092757, indexed in Pubmed: 32927735.

20. Luo X, Liao Q, Shen $Y$, et al. Vitamin D Deficiency Is Associated with COVID-19 Incidence and Disease Severity in Chinese People [corrected]. J Nutr. 2021; 151(1): 98-103, doi: 10.1093/jn/nxaa332, indexed in Pubmed: 33188401.

21. Vassiliou AG, Jahaj E, Pratikaki $M$, et al. Low 25-Hydroxyvitamin D Levels on Admission to the Intensive Care Unit May Predispose COVID-19 Pneumonia Patients to a Higher 28-Day Mortality Risk: A Pilot Study on a Greek ICU Cohort. Nutrients. 2020; 12(12), doi: 10.3390/nu12123773, indexed in Pubmed: 33316914.

22. Jain A, Chaurasia R, Sengar NS, et al. Analysis of vitamin D level among asymptomatic and critically ill COVID-19 patients and its correlation with inflammatory markers. Sci Rep. 2020; 10(1): 20191, doi: 10.1038/ s41598-020-77093-z, indexed in Pubmed: 33214648.

23. Jevalikar G, Mithal A, Singh A, et al. Lack of Association of Baseline 25-Hydroxyvitamin $D$ Levels and Cholecalciferol Treatment With 
Disease Severity and Mortality in Indian Patients Hospitalized for Covid-19. , doi: 10.21203/rs.3.rs-129238/v1.

24. Tehrani S, Khabiri N, Moradi H, et al. Evaluation of vitamin D levels in COVID-19 patients referred to Labafinejad hospital in Tehran and its relationship with disease severity and mortality. Clin Nutr ESPEN. 2021; 42: 313-317, doi: 10.1016/j.clnesp.2021.01.014, indexed in Pubmed: 33745598.

25. Carpagnano GE, Di Lecce V, Quaranta VN, et al. Vitamin D deficiency as a predictor of poor prognosis in patients with acute respiratory failure due to COVID-19. J Endocrinol Invest. 2021; 44(4): 765-771, doi: 10.1007/s40618-020-01370-x, indexed in Pubmed: 32772324.

26. Cereda E, Bogliolo L, Klersy C, et al. NUTRI-COVID19 IRCCS San Matteo Pavia Collaborative Group. Vitamin D 250H deficiency in COVID-19 patients admitted to a tertiary referral hospital. Clin Nutr. 2021; 40(4): 2469-2472, doi: 10.1016/j.clnu.2020.10.055, indexed in Pubmed: 33187772.

27. Ricci A, Pagliuca A, D'Ascanio M, et al. Circulating Vitamin D levels status and clinical prognostic indices in COVID-19 patients. Respir Res. 2021; 22(1): 76, doi: 10.1186/s12931-021-01666-3, indexed in Pubmed: 33658032.

28. Hernández JL, Nan D, Fernandez-Ayala M, et al. Vitamin D Status in Hospitalized Patients with SARS-CoV-2 Infection. J Clin Endocrinol Metab. 2021; 106(3): e1343-e1353, doi: 10.1210/clinem/dgaa733, indexed in Pubmed: 33159440.

29. Demir M, Demir F, Aygun H. Vitamin D deficiency is associated with COVID-19 positivity and severity of the disease. J Med Virol. 2021; 93(5): 2992-2999, doi: 10.1002/jmv.26832, indexed in Pubmed: 33512007.

30. Karahan S, Katkat F. Impact of Serum 25(OH) Vitamin D Level on Mortality in Patients with COVID-19 in Turkey. J Nutr Health Aging. 2021; 25(2): 189-196, doi: 10.1007/s12603-020-1479-0, indexed in Pubmed: 33491033.

31. Tuncay ME, Gemcioglu E, Kayaaslan B, et al. A notable key for estimating the severity of COVID-19: 25-hydroxyvitamin D status. Turkish Journal of Biochemistry. 2021; 46(2): 167-172, doi: 10.1515/ tjb-2020-0423.

32. Panagiotou G, Tee SuA, Ihsan Y, et al. Low serum 25-hydroxyvitamin $D(25[0 H] D)$ levels in patients hospitalized with COVID-19 are associated with greater disease severity. Clin Endocrinol (0xf). 2020; 93(4): 508-511, doi: 10.1111/cen.14276, indexed in Pubmed: 32621392.

33. Baktash V, Hosack T, Patel N, et al. Vitamin D status and outcomes for hospitalised older patients with COVID-19. Postgrad Med J. 2021; 97(1149): 442-447, doi: 10.1136/postgradmedj-2020-138712, indexed in Pubmed: 32855214.

34. Orchard L, Baldry M, Nasim-Mohi M, et al. Vitamin-D levels and intensive care unit outcomes of a cohort of critically ill COVID-19 patients. Clin Chem Lab Med. 2021; 59(6): 1155-1163, doi: 10.1515/ cclm-2020-1567, indexed in Pubmed: 33554566.

35. Mazziotti G, Lavezzi E, Brunetti A, et al. Humanitas COVID19 Task Force. Vitamin $D$ deficiency, secondary hyperparathyroidism and respiratory insufficiency in hospitalized patients with COVID-19.
J Endocrinol Invest. 2021 [Epub ahead of print], doi: 10.1007/s40618021-01535-2, indexed in Pubmed: 33666876.

36. Clements DN, Bruce G, Ryan JM, et al. Effects of surgery on free and total 25 hydroxyvitamin $D$ concentrations in dogs. J Vet Intern Med. 2020; 34(6): 2617-2621, doi: 10.1111/jvim.15933, indexed in Pubmed: 33179819.

37. Waldron JL, Ashby HL, Cornes MP, et al. Vitamin D: a negative acute phase reactant. J Clin Pathol. 2013; 66(7): 620-622, doi: 10.1136/ jclinpath-2012-201301, indexed in Pubmed: 23454726.

38. Reid D, Toole BJ, Knox S, et al. The relation between acute changes in the systemic inflammatory response and plasma 25-hydroxyvitamin D concentrations after elective knee arthroplasty. Am J Clin Nutr. 2011; 93(5): 1006-1011, doi: 10.3945/ajcn.110.008490, indexed in Pubmed: 21411617.

39. Louw JA, WerbeckA, Louw ME, et al. Blood vitamin concentrations during the acute-phase response. Crit Care Med. 1992; 20(7): 934-941, doi: 10.1097/00003246-199207000-00007, indexed in Pubmed: 1617986.

40. Barth JH, Field HP, Mather AN, et al. Serum 25 hydroxy-vitamin $D$ does not exhibit an acute phase reaction after acute myocardial infarction. Ann Clin Biochem. 2012; 49(Pt 4): 399-401, doi: 10.1258/ acb.2011.011195, indexed in Pubmed: 22543926.

41. Bang UC, Novovic $S$, Andersen AM, et al. Variations in serum 25-hydroxyvitamin D during acute pancreatitis: an exploratory longitudinal study. Endocr Res. 2011; 36(4): 135-141, doi: 10.3109/07435800.2011.554937, indexed in Pubmed: 21973232.

42. Bertoldo F, Pancheri S, Zenari S, et al. Serum 25-hydroxyvitamin $D$ levels modulate the acute-phase response associated with the first nitrogen-containing bisphosphonate infusion. J Bone Miner Res. 2010; 25(3): 447-454, doi: 10.1359/jbmr.090819, indexed in Pubmed: 20200999.

43. Krishnan A, Ochola J, Mundy J, et al. Acute fluid shifts influence the assessment of serum vitamin $D$ status in critically ill patients. Crit Care. 2010; 14(6): R216, doi: 10.1186/cc9341, indexed in Pubmed: 21110839.

44. Quraishi SA, Camargo CA. Vitamin D in acute stress and critical illness. Curr Opin Clin Nutr Metab Care. 2012; 15(6): 625-634, doi: 10.1097/ MC0.0b013e328358fc2b, indexed in Pubmed: 23075939.

45. McMillan DC, Maguire D, Talwar D. Relationship between nutritional status and the systemic inflammatory response: micronutrients. Proc Nutr Soc. 2019; 78(1): 56-67, doi: 10.1017/S0029665118002501, indexed in Pubmed: 30220267.

46. Zgaga L, Laird E, Healy M. 25-Hydroxyvitamin D Measurement in Human Hair: Results from a Proof-of-Concept study. Nutrients. 2019; 11(2), doi: 10.3390/nu11020423, indexed in Pubmed: 30781610.

47. MacDonell SO, Miller JC, Harper MJ, et al. A comparison of methods for adjusting biomarkers of iron, zinc, and selenium status for the effect of inflammation in an older population: a case for interleukin 6. Am J Clin Nutr. 2018; 107(6): 932-940, doi: 10.1093/ajcn/nqy052, indexed in Pubmed: 29767675.

48. Celikbilek A. Vitamin D axis status and the severity of COVID-19. J Med Virol. 2021; 93(7): 4085, doi: 10.1002/jmv.26920, indexed in Pubmed: 33666241. 
49. Powe CE, Evans MK, Wenger J, et al. Vitamin D-binding protein and vitamin $D$ status of black Americans and white Americans. N Engl J Med. 2013; 369(21): 1991-2000, doi: 10.1056/NEJMoa1306357, indexed in Pubmed: 24256378.
50. Butler-Laporte G, Nakanishi T, Mooser V, et al. Vitamin D and COVID-19 susceptibility and severity in the COVID-19 Host Genetics Initiative: A Mendelian randomization study. PLoS Med. 2021; 18(6): e1003605, doi: 10.1371/journal.pmed.1003605, indexed in Pubmed: 34061844. 